\title{
Extracting Residual NMR Coupling Constants From Electrically Aligned Liquids
}

\author{
Scott A. Riley and Matthew P. Augustine* \\ Department of Chemistry, One Shields Avenue, University of California, Davis, California 95616
}

Received: January 5, 2000

\begin{abstract}
Molecular alignment by an electric field is used to reintroduce quadrupolar couplings into the nuclear magnetic resonance spectrum of polar liquids. By interleaving a pulsed electric field with rf pulse sequences in a controlled way, spectral simplification is introduced by correlating isotropic chemical shifts with residual anisotropic couplings in a two-dimensional fashion. The success of these experiments is facilitated by combining rapid $\pi$ rf pulsing with multiple quantum filtration to minimize molecular motion effects on the measured signal. An analysis of the observed coupling constants and their relation to molecular structure are provided.
\end{abstract}

\section{Introduction}

The ability of nuclear magnetic resonance (NMR) spectroscopy to provide detailed information about molecular structure and dynamics has led to a wide range of applications in chemistry, biochemistry, and materials science. ${ }^{1}$ Considering the rapidly growing number of molecular structures $(>1500)$ determined by NMR techniques alone ${ }^{2}$ one might believe the need for technological advances within this already mature field to be decreasing. Indeed, analysis of a fixed set of one-, two-, and sometimes three-dimensional NMR spectra often provides an adequate picture of molecular structure in liquids. ${ }^{3}$ When tertiary or quaternary structure is studied, the critical parameter extracted from these measurements is the through-space distance between nuclei, typically manifested in a relaxation rate and monitored as a change in peak intensity due to the nuclear Overhauser effect (NOE). ${ }^{4}$ The chief limitation in determining molecular structure in liquids using the NOE is that the throughspace distance between nuclei $i$ and $j, r_{i, j}$, comes into the measured rates via the square of the homo- or heteronuclear dipolar coupling as $1 / r_{i, j}{ }^{6}$, forcing reliable distance estimates to be less than $5 \AA$ A. ${ }^{5}$ Several methods have been developed to combat this length scale problem. Instead of measuring a distance dependent rate, techniques such as mechanical sample orientation, ${ }^{6}$ single crystal NMR, ${ }^{7}$ and solid-state NMR on powdered samples ${ }^{8}$ strive to determine the dipolar coupling $D_{i, j}$ directly. Since $D_{i, j}$ is proportional to $1 / r_{i j}{ }^{39}$ much longer distances can be measured. In the case of biological samples, one might argue that these experiments do not truly reflect the native liquid environment of the protein or nucleic acid under investigation. For this reason and others Tolman and Prestegard, ${ }^{10}$ Tjandra and Bax, ${ }^{11}$ and others ${ }^{12,13}$ have suggested using residual dipolar couplings in solutions of samples having an anisotropic magnetic susceptibility $\Delta \chi$ as an alternative means of obtaining distance estimates. Here $\Delta \chi$ interacts with the DC polarizing field, typically in excess of $11 \mathrm{~T}$, to partially align molecules either parallel or perpendicular to the applied magnetic field, depending on the sign of $\Delta \chi \cdot{ }^{14}$ It is this macroscopic molecular alignment that reintroduces anisotropic spectral features in the form of line shifts and splittings into the liquid state NMR spectrum. An estimate of the magnitude of this alignment can be obtained by first considering a proton

* Corresponding author. E-mail: augustin@chem.ucdavis.edu. spin pair rigidly held parallel to the external magnetic field and separated by $1 \AA$ such that $D_{i, j}=120.1 \mathrm{kHz} .{ }^{9}$ In the absence of alignment, i.e. for an isotropic liquid, the average value $\left\langle D_{i, j}\right\rangle$ $=0$; however, for molecules with nonzero $\Delta \chi$ values, $\left\langle D_{i, j}\right\rangle$ is typically $1-10 \mathrm{~Hz}$ for the same proton spin pair, yielding an alignment factor of $10 \mathrm{~Hz} / 120.1 \mathrm{kHz}=8 \times 10^{-5}$.

This article presents results obtained from exploration of an alternative means of molecular alignment in liquid phase. Here, both continuous and pulsed electric fields are used to align polar molecules while NMR is used to examine correlations between isotropic and electrically induced anisotropic spectral effects. In comparison to magnetic alignment, this approach relies on the interaction between an electric dipole moment, $p$, and an applied electric field, $E$, and has the potential to refine highresolution molecular structures by NMR via measurement of residual structurally dependent anisotropic coupling constants like the dipolar interaction. Most polar molecules have dipole moments between 1 and $5 \mathrm{D}$. As pointed out by Sears and Hahn, ${ }^{15}$ MacLean, ${ }^{16}$ and others ${ }^{17,18}$ in terms of parameters introduced above, for a typical applied electric field of $E_{\text {app }}=$ $50 \mathrm{kV} / \mathrm{cm}$, alignment factors about an order of magnitude larger than those anticipated for magnetic alignment can be expected. Consequently, distance dependent residual couplings will be more pronounced in an electric field, e.g. $\left\langle D_{i, j}\right\rangle \approx 10-100 \mathrm{~Hz}$ for the same two protons separated by $1 \AA$ mentioned above.

It is useful to comment on measurement of these residual couplings and their separation from isotropic spectra. Both the magnetic and electric strategies require measurements at several different field strengths in order to differentiate between scalar spectral effects and field dependent molecular alignment effects. In the case of magnetic alignment this immediately requires at least three high-field instruments, typically NMR spectrometers operating at proton Larmor frequencies of 500, 600, and 800 $\mathrm{MHz}$. Electrical alignment, on the other hand, simply uses one NMR spectrometer, since measurements in electric fields of different values may easily be accomplished using a variable voltage power supply. Both methods necessarily increase the complexity of the measured spectrum, which in the case of macromolecules can make peak assignments and thus structure determination impossible. Consequently, sequences have been designed to separate spectral information for magnetically aligned samples into multiple dimensions. These sequences should in principle work for molecular alignment in a continuous 
$E$ field; however, increased molecular motion on the time scale of the NMR experiment initiated by electrode charge injection washes out the effect. For this reason, new sequences have been developed to compensate for these motional effects on the observed NMR signal. Electrical alignment also has the added advantage of being gated. At any time within an NMR pulse sequence one can align molecules at will-an advantage not shared by magnetic alignment due to the unavailability of switchable multitesla magnetic fields. This luxury can be exploited in the design of new correlation experiments to extract structural information.

Although the pulse sequences presented here are applicable to spin systems displaying either residual dipolar $\hbar D_{i, j}$ or quadrupolar $\hbar Q_{i}$ couplings, discussion here is limited only to an $I=1$ test system. Since $Q_{i}$ is typically greater than $D_{i, j}$ for $I>1 / 2$ nuclei in noncentrosymmetric electronic environments, the residual quadrupolar coupling constant $\left\langle Q_{i}\right\rangle$ is a more sensitive measure of electrically induced anisotropy in liquids by NMR.

\section{Experimental Section}

Only relevant characteristics of the experimental setup are highlighted here because all details concerning the home-built NMR probe head, spectrometer, electric field cell, and associated electronics are surveyed in detail elsewhere. ${ }^{19}$ The $3.62 \mathrm{~mm}$ long $\times 11 \mathrm{~mm}$ diameter cylindrical sample is housed between two $0.5 \mathrm{~mm}$ thick $\times 12 \mathrm{~mm}$ diameter copper electrodes. Ion purification membranes obtained from Ionics, Inc. separate the electrodes from the main sample volume for in situ ionic purification of the sample. An electric field is launched across the sample by connecting the electrodes via high-voltage cable to a TTL-controlled Kilovac K62C-841 $25 \mathrm{kV}$ relay and finally to a Bertan 210-50R $50 \mathrm{kV}$ power supply. Values of the applied electric field, $E_{\text {app }}$, reported here are calculated by dividing the voltage applied to the electrodes by the electrode spacing in order to remain consistent with previous electrical alignment studies. Voltage rise times upon gating of the relay are less than $1 \mathrm{~ms}$. Both electrodes and sample cell are contained within a Helmholtz rf coil that is housed within the NMR probe head and tuned to the $45.37 \mathrm{MHz}$ deuterium ${ }^{2} \mathrm{H}$ Larmor frequency in a $6.95 \mathrm{~T}$ magnetic field. Proper shimming of the static magnetic field yields ${ }^{2} \mathrm{H}$ NMR spectral line widths of $7 \mathrm{~Hz}$. All electronics are controlled by a Tecmag Orion pulse programmer. Several samples have been examined with this setup; however, due to the large volume of literature concerning perdeuterated nitrobenzene $\mathrm{C}_{6} \mathrm{D}_{5} \mathrm{NO}_{2}$ in electric fields and because the molecule contains multiple quadrupolar nuclei, the discussion here is limited to this molecule. Samples of perdeuterated nitrobenzene $(99.5 \%)$ obtained from Aldrich required several hours of electropurification in the sample cell via the ion purification membranes accomplished by gradually increasing the field to operating values of $50-60 \mathrm{kV} / \mathrm{cm}$ until a sufficiently low conductivity of $\sigma \approx 8 \times 10^{-11}(\Omega \mathrm{cm})^{-1}$ was reached. A typical sample could be used for several days of experiments before electrical breakdown through the liquid occurred. All semiempirical structure calculations were accomplished using PC Spartan Pro Version 1.0 obtained from Wavefunction, Inc.

\section{NMR Spectra of Electrically Aligned Molecules}

Basic Considerations. Before considering the design of pulse sequences and expected results, it is useful to recall how molecular alignment modifies observable NMR spectra. This knowledge can then be used to judge the effectiveness and accuracy of pulse sequences interleaved with static and pulsed electric fields for molecular structure determination. In the special case of $I=1{ }^{2} \mathrm{H}$ nuclei, there will be two allowed $\Delta m$ $= \pm 1$ transitions per site $i$. These frequencies

$$
\omega_{i}=\sigma_{i} \pm\left\langle 3 \cos ^{2} \theta_{i}-1\right\rangle Q_{i}=\sigma_{i} \pm\left\langle Q_{i}\right\rangle
$$

depend not only on the isotropic chemical shift $\sigma_{i}$, but also on $Q_{i}$ and the angle $\theta_{i}$ between the laboratory axis system set by the direction of the magnetic $B_{0}$ and electric fields and the principal axis system (PAS) of the second rank quadrupolar coupling tensor, here chosen to be symmetric with $\eta=0$. A good approximation to the residual coupling $\left\langle Q_{i}\right\rangle$ in eq 1 that neglects alignment cooperativity due to interparticle correlations can be determined from the Boltzmann average

$$
\left\langle 3 \cos ^{2} \theta_{i}-1\right\rangle=\frac{\iint\left(3 \cos ^{2} \theta_{i}-1\right) \mathrm{P}\left(\theta_{i}\right) \sin \theta_{i} \mathrm{~d} \theta_{i} \mathrm{~d} \phi_{i}}{\iint \mathrm{P}\left(\theta_{i}\right) \sin \theta_{i} \mathrm{~d} \theta_{i} \mathrm{~d} \phi_{i}}
$$

The consequence of the integral over the phase angle $\phi_{i}$ is to nullify all angular contributions to $\left\langle Q_{i}\right\rangle$ that depend on $\mathrm{e}^{ \pm i \phi_{i}}$. In the special case where the PAS and the direction of the molecular dipole moment, $\mathbf{p}$, coincide, this average reduces to $2(\mathrm{pE} / \mathrm{kT})^{2} / 15$ in the high-temperature limit where $\mathrm{P}\left(\theta_{i}\right)=\mathrm{e}^{-\mathbf{p} \cdot \mathbf{E} / k T}$ $\approx 1-(p E / k T) \cos \theta_{i}$. In most interesting cases, the direction of the PAS and $\mathbf{p}$ are not degenerate and an additional rotation from the PAS to the direction of $\mathbf{p}$ by the set of Euler angles $\left(\alpha_{i}, \beta_{i}, \gamma_{i}\right)$ must be accomplished prior to averaging over the angles $\left(\alpha_{i}^{\prime}, \beta_{i}^{\prime}, \gamma_{i}^{\prime}\right)$ relating $\mathbf{p}$ to the laboratory frame of reference. Accounting for this rotation in a planar system like $\mathrm{C}_{6} \mathrm{D}_{5} \mathrm{NO}_{2}$, where it is safe to consider the PAS of the quadrupolar coupling tensor to lie along the $\mathrm{C}-\mathrm{D}$ bond, ${ }^{20}$ recasts the residual coupling in eq 1 as

$$
\left\langle Q_{i}\right\rangle=\frac{2}{15}\left(\frac{p E}{k T}\right)^{2}\left(\frac{3 \cos ^{2} \beta_{i}-1}{2}\right) Q_{i}
$$

in the high-temperature limit, where $\beta_{i}$ is the angle between the $\mathrm{C}-\mathrm{D}$ bond direction and $\mathbf{p}$. In the gas phase, the alignment factor proportional to $2(p E / k T)^{2} / 15$ is reasonably well-approximated with the gas-phase dipole moment $p=p_{\text {gas }}$ and the applied value of the electric field $E=E_{\text {app }}$ set by the geometry of and voltage applied to the plates. In condensed phase described by a dielectric constant $\epsilon$ and index of refraction $n$, this approximation breaks down. The effective dipole moment $p$ and field $E$ are then scaled to the Onsager ${ }^{21}$ values of

$$
p=\frac{(2 \epsilon+1)\left(n^{2}+2\right)}{3\left(2 \epsilon+n^{2}\right)} p_{\text {gas }}
$$

and

$$
E=\frac{3 \epsilon}{2 \epsilon+1} E_{\text {app }}
$$

For $\mathrm{C}_{6} \mathrm{H}_{5} \mathrm{NO}_{2}$ with $p_{\text {gas }}=4.2 \mathrm{D}, \epsilon=35$, and $n=1.56$ in an $E_{\text {app }}=55 \mathrm{kV} / \mathrm{cm}$ electric field, eqs 4 and 5 yield $p=6.08 \mathrm{D}$ and $E=81.3 \mathrm{kV} / \mathrm{cm}$. These values can be used to determine the size of the alignment factor in eq 3 as $2(p E / k T)^{2} / 15=2.14$ $\times 10^{-4}$. Coupling this calculation with eqs 1 and 3 and sitespecific values for the chemical shifts $\sigma_{i}$, quadrupolar couplings $Q_{i}$, and C-D bond angles $\beta_{i}$ allows a reliable prediction of the ${ }^{2} \mathrm{H}$ NMR spectrum corresponding to $\mathrm{C}_{6} \mathrm{D}_{5} \mathrm{NO}_{2}$ in an $E_{\text {app }}=55$ $\mathrm{kV} / \mathrm{cm}$ electric field. 


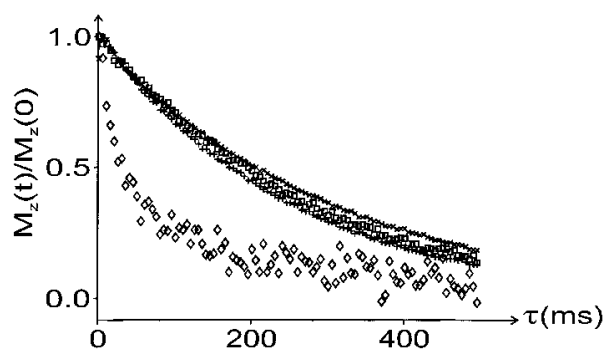

Figure 1. Comparison of longitudinal relaxation times for all sites in $\mathrm{C}_{6} \mathrm{D}_{5} \mathrm{NO}_{2}$ with $E_{\text {app }}=0 \mathrm{kV} / \mathrm{cm}(+$ and $\square)$ and $E_{\text {app }}=40 \mathrm{kV} / \mathrm{cm}(\times$ and $\diamond)$ using the inversion recovery experiment $(+$ and $\times)$ and the stimulated echo sequence $(\square$ and $\diamond$ ) with $\tau=200 \mu$ s. The data corresponding to the inversion recovery experiment was shifted to appear as a decay from +1 at $t=0$. The magnetization decay rate observed using the inversion recovery experiment with $E_{\text {app }}=0 \mathrm{kV} /$ $\mathrm{cm}$ and $40 \mathrm{kV} / \mathrm{cm}$ and the stimulated echo sequence with $E_{\text {app }}=0$ $\mathrm{kV} / \mathrm{cm}$ is $3.8 \mathrm{~s}^{-1}$. Increasing $E_{\text {app }}$ to $40 \mathrm{kV} / \mathrm{cm}$ for the stimulated echo sequence decreases the decay rate to $13.0 \mathrm{~s}^{-1}$.

Accompanying the three distinct chemical sites in $\mathrm{C}_{6} \mathrm{D}_{5} \mathrm{NO}_{2}$ (ortho, meta, and para) are the separate chemical shifts $\sigma_{o}, \sigma_{m}$, and $\sigma_{p}$ as well as the quadrupolar coupling constants $Q_{o}, Q_{m}$, and $Q_{p}$ and the $\mathrm{C}-\mathrm{D}$ bond angles $\beta_{o}, \beta_{m}$, and $\beta_{p}$. Of particular interest here are the $Q_{i}$ and $\beta_{i}$ values that can be estimated in the $\eta=0$ limit from liquid crystal NMR data. Typical values of these parameters are $Q_{o}=185 \mathrm{kHz}, Q_{m}=210 \mathrm{kHz}, Q_{p}=$ $185 \mathrm{kHz}, \beta_{o}=58.3^{\circ}, \beta_{m}=118.9^{\circ}$, and $\beta_{p}=180^{\circ} .{ }^{20}$ Using eq 3 in eq 1 with $E$ and $p$ defined above indicates that the deuteron para to the $-\mathrm{NO}_{2}$ group having its PAS parallel to $\mathbf{p}$ will have the full residual coupling $\left\langle Q_{p}\right\rangle=40 \mathrm{~Hz}$ since $\beta_{p}=180^{\circ}$. The values for $\left\langle Q_{i}\right\rangle$ corresponding to the meta and ortho sites are scaled by 0.15 and 0.09 , respectively, to give $\left\langle Q_{m}\right\rangle=7 \mathrm{~Hz}$ and $\left\langle Q_{o}\right\rangle=4 \mathrm{~Hz}$, because $\beta_{m}=118.9^{\circ}$ and $\beta_{o}=58.3^{\circ}$ in eq 3 . Using these estimates of $\left\langle Q_{i}\right\rangle$ for $i=$ ortho, meta, and para allows the spectrum as a function of $E_{\text {app }}$ to be uniquely determined. When $E_{\text {app }}=0 \mathrm{kV} / \mathrm{cm}, E=0 \mathrm{kV} / \mathrm{cm}$ giving $\left\langle Q_{i}\right\rangle=0$. Therefore, a three-peak NMR spectrum in a 2:1:2 intensity ratio should be observed, reflecting the different shifts $\sigma_{i}$ of the two ortho and meta deuterons and the lone para deuteron in $\mathrm{C}_{6} \mathrm{D}_{5} \mathrm{NO}_{2}$. Switching the electric field on causes all resonances to split as a function of $E_{\text {app }}{ }^{2}$ with the splitting for the para deuteron always larger than those for the ortho and meta peaks. Indeed, for $E_{\text {app }}$ $=55 \mathrm{kV} / \mathrm{cm}$, eq 1 with eq 3 indicates that a six-peak spectrum should be present with the splitting for the para deuteron being $2\left\langle Q_{p}\right\rangle=80 \mathrm{~Hz}$, roughly $80 / 14 \approx 80 / 8=10$ times larger than that for the two meta and ortho peaks.

Development of Pulse Sequences. The basic building blocks of two-dimensional (2D) pulse sequences designed to correlate electric field induced residual anisotropic couplings with isotropic spectra are multiple $\pi \mathrm{rf}$ pulses and double quantum filtration. ${ }^{4}$ The multiple $\pi$ rf pulses serve to minimize the effects of electrically induced convection on the measured NMR signal, as was demonstrated by Carr and Purcell for the case of molecular diffusion. ${ }^{22}$ The double quantum filter selects only the $E$ dependent spectral frequencies in the signal, $\left\langle Q_{i}\right\rangle$ or equivalently $\left\langle D_{i, j}\right\rangle$. The repetition rate of the $\pi$ pulses is set by the time scale of motion, here approximately $10 \mathrm{~ms}$ as can be seen in separate measurements of the relaxation rate shown in Figure 1 . The + and $\times$ symbols designate the recovery of inverted magnetization to the equilibrium $+z$ direction using the inversion recovery technique while the $\square$ and $\diamond$ symbols label the magnetization decay monitored with the stimulated echo sequence. Regardless of the state of the electric field, $E_{\text {app }}$ $=0 \mathrm{kV} / \mathrm{cm}(+)$ or $E_{\text {app }}=40 \mathrm{kV} / \mathrm{cm}(\times)$, the inversion recovery experiment, which is independent of molecular position in a a)
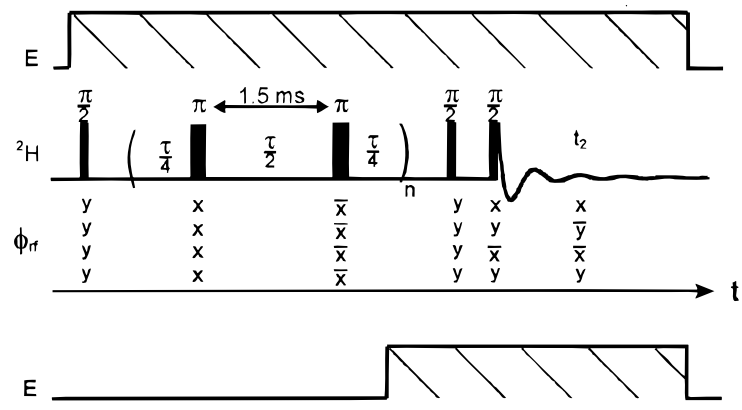

b)

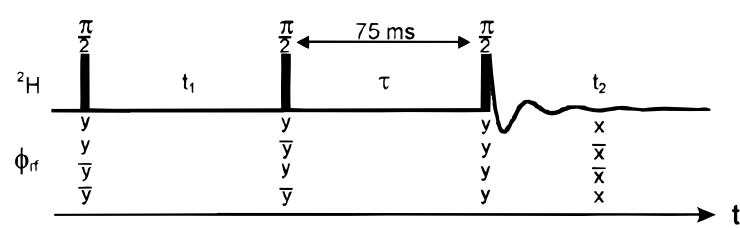

c)

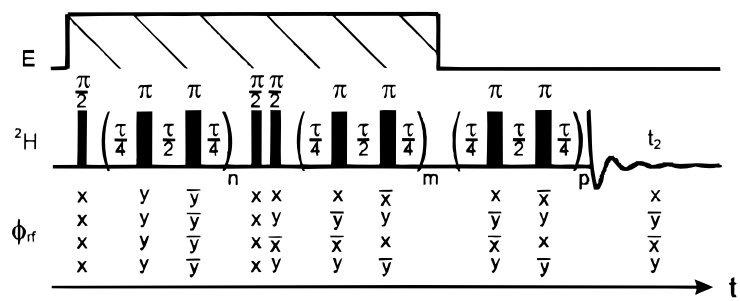

Figure 2. Pulse sequences used in this study showing the rf pulse phase as well as the timing of electric field gating and rf pulses. The sequence in panel a is used to correlate the usual $1 \mathrm{D}^{2} \mathrm{H}$ NMR spectrum obtained in a DC electric field that displays peaks having both electrically dependent and independent frequencies with electrically dependent effects. As discussed in the text, the $\pi$ rf pulses minimize motional effects due to electrical convection in the resulting spectra, while the pair of $\pi / 2 \mathrm{rf}$ pulses select for electrically induced information. Time increments in the indirect dimension are accomplished by incrementing $n$ such that $t_{1}=n \tau$. The pulse sequence in panel b correlates an isotropic spectrum obtained during the time $t_{1}$ with a spectrum having contributions from both isotropic and anisotropic effects measured as a function of the time $t_{2}$ following gating the electric field on during $\tau$. A combination of multiple $\pi \mathrm{rf}$ pulses, double quantum filtration, and pulsed electric fields in panel $\mathrm{c}$ can be used to truly correlate an isotropic $E_{\text {app }}=0 \mathrm{kV} / \mathrm{cm}$ spectrum observed during the time $t_{2}$ with an anisotropic spectrum measured as a function of $t_{1}$.

slightly inhomogeneous magnetic field, gives nearly the same decay rate. On the other hand, the decay rate from the stimulated echo sequence, which strongly reflects molecular motion, even when high-resolution line widths less than $7 \mathrm{~Hz}$ are used, indicates a pronounced increase in the magnetization decay rate when $E_{\text {app }}$ is increased from $0 \mathrm{kV} / \mathrm{cm}(\square)$ to $40 \mathrm{kV} / \mathrm{cm}(\diamond)$. The increase in decay rate for the stimulated echo from $3.8 \mathrm{~s}^{-1}$ at $E_{\text {app }}=0 \mathrm{kV} / \mathrm{cm}$ to $13.0 \mathrm{~s}^{-1}$ at $E_{\text {app }}=40 \mathrm{kV} / \mathrm{cm}$, combined with both the observation that the standard $\pi / 2-t_{1}-\pi$ spin echo is appreciably attenuated for $t_{1}=20 \mathrm{~ms}$ when $E_{\text {app }}>20 \mathrm{kV} /$ $\mathrm{cm}$ and magnetic resonance imaging investigations of electrical convection made elsewhere, ${ }^{19}$ indicates that the time scale of motion is tens of milliseconds.

The pulse sequences in Figure 2 incorporate both multiple $\pi$ rf pulses and double quantum filtration. Each sequence shown here includes the timing of the electric field switching, marked as $E$ as well as the phases of the rf pulses, labeled as $\phi_{\mathrm{rf}}$. Not included in this figure are the phases of the first rf pulse with respect to $t_{1}$. In all 2D measurements time proportional phase incrementation is used for complex data acquisition during the period $t_{1}$. The five $\mathrm{rf}$ pulses preceding the detection event in the pulse sequence in Figure 2a serve to eliminate the isotropic chemical shift $\sigma_{i}$ from the spectrum, leaving quadrupolar 
information $\left\langle Q_{i}\right\rangle$ obtained during $t_{1}=n \tau$ intact. It should be mentioned at this point that the notation $t_{1}=n \tau$ means that $n$ is allowed to vary over all integer values between and including 1 and 80. In terms of the sequence in Figure 2a, each successive $t_{1}$ point contains an extra set of $\pi$ pulses. The two $\pi / 2 \mathrm{rf}$ pulses immediately prior to detection with respect to $t_{2}$ are phasecycled, as shown in the group of phases labeled as $\phi_{\mathrm{rf}}$ in Figure $2 \mathrm{a}$, to behave as a double quantum filter. By operating in this way one can correlate electrical effects with the usual 1D NMR spectrum observed in a CW electric field.

By interleaving a pulsed electric field with an rf pulse sequence in a controlled way as shown in the sequences in Figure 2b,c, true correlations between electric field dependent anisotropic spectral effects and isotropic spectral effects, as well as combinations of the two can be generated. Several variations of sequences involving gated electric fields can be imagined; however, perhaps the simplest version is shown in Figure $2 b$. Here the directly measured spectrum as a function of $t_{2}$ reflects both chemical shifts $\sigma_{i}$ and electrically induced quadrupolar couplings $\left\langle Q_{i}\right\rangle$, and for $\mathrm{C}_{6} \mathrm{D}_{5} \mathrm{NO}_{2}$ will yield a six-peak spectrum. The indirectly detected spectrum as a function of $t_{1}$ contains only chemical shifts, because the electric field is gated off during the time period $t_{1}$. The purpose of the central $\pi / 2 \mathrm{rf}$ pulse in the sequence shown in Figure $2 \mathrm{~b}$ is to store a component of the frequency-encoded magnetization with respect to $t_{1}$ along the $z$ direction. The delay $\tau=75 \mathrm{~ms}$ was chosen to ensure that a reproducible electric field is established within the sample. Application of the final $\pi / 2 \mathrm{rf}$ pulse rotates the magnetization stored along the $z$ direction before $\tau$ into the transverse plane prior to detection during the period $t_{2}$. Phase cycling the rf pulses and receiver as shown in the line labeled as $\phi_{\mathrm{rf}}$ in Figure $2 b$ eliminates unwanted signals due to any single rf pulse alone and selects only for the stimulated echo.

The more advanced variant of these $2 \mathrm{D}$ correlation sequences is shown in Figure 2c. By combining the multiple rf $\pi$ pulsing, double quantum filtration, and pulsed electric fields used in the pulse sequences in Figure 2a,b one can generate and measure a true correlation between the usual $E_{\text {app }}=0 \mathrm{kV} / \mathrm{cm}$ isotropic liquids spectrum and electrically dependent anisotropic spectral effects alone. The indirect dimension between the first $\pi / 2 \mathrm{rf}$ pulse and the two $\pi / 2 \mathrm{rf}$ pulses comprising the double quantum filter in the sequence in Figure $2 \mathrm{c}$ is identical to that shown in Figure 2a. Here, $n$ is incremented from 1 to 64 in order to increase $t_{1}$ as $n \tau$. The purpose of the second group of $\pi$ pulses in Figure $2 \mathrm{c}$ is to allow the anisotropic effects selected during $t_{1}$ to rephase from unobservable antiphase magnetization into observable single quantum magnetization while suspending the evolution of all chemical shifts. One has the freedom to increment this second set of $\pi$ pulses along with the first; however, $m$ was held fixed in the measurements reported here. The third set of $\pi$ pulses is used only to suspend chemical shift evolution while the sample and electronics recover from the gating of the electric field pulse. This serves to simplify the interpretation of data accumulated during the indirect evolution period $t_{1}=n \tau$. In contrast to the sequences shown in Figure 2a,b, detection during $t_{2}$ followed by Fourier transformation yields an isotropic $E_{\text {app }}=0 \mathrm{kV} / \mathrm{cm}$ spectrum.

\section{Results and Discussion}

The direct $t_{2}$ dimension of the pulse sequence in Figure $2 \mathrm{a}$ corresponds to free precession of the magnetization in the presence of an electric field. The projection shown at the top of Figure $3 \mathrm{a}$ for $E_{\mathrm{app}}=55 \mathrm{kV} / \mathrm{cm}$ shows the anticipated sixpeak one-dimensional ${ }^{2} \mathrm{H}$ NMR spectrum of $\mathrm{C}_{6} \mathrm{D}_{5} \mathrm{NO}_{2}$ resulting
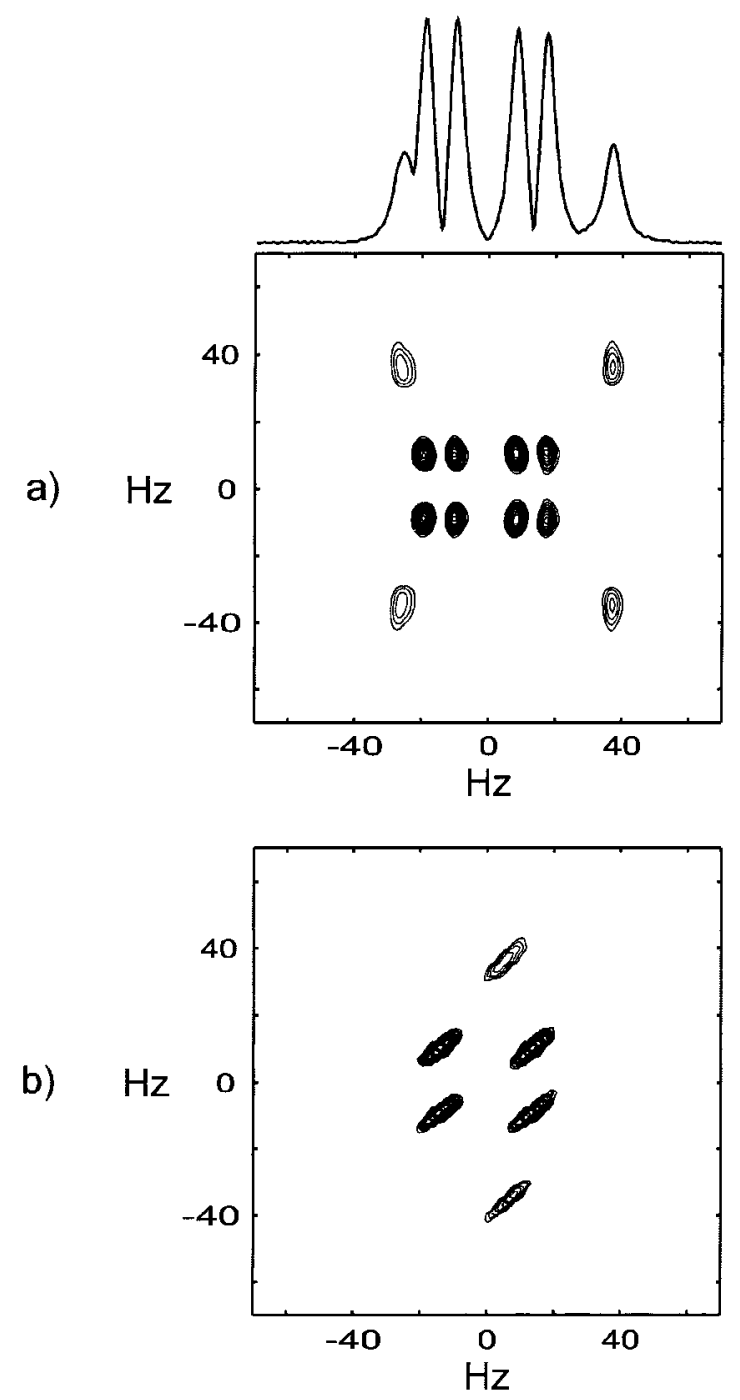

Figure 3. Two-dimensional ${ }^{2} \mathrm{H}$ NMR spectra correlating isotropic and anisotropic spectral effects in a continuous DC electric field of $E_{\text {app }}=$ $55 \mathrm{kV} / \mathrm{cm}$. The spectrum in panel a shown in absolute value mode corresponds to Fourier transformation as a function of both $t_{1}$ and $t_{2}$ of the data collected using the pulse sequence in Figure 2a. The onedimensional spectrum at the top is a sum projection of the horizontal slices in panel $\mathrm{a}$. The spectrum in panel $\mathrm{b}$ is produced by application of a shearing transformation to the positively phased peaks of the raw spectral data in panel a.

from Fourier transformation of the free precession signal with respect to $t_{2}$ in the sequence in Figure 2a. Specifically, the two outer peaks correspond to the lone para deuteron while the two other groups of peaks centered at $+15 \mathrm{~Hz}$ and $-17 \mathrm{~Hz}$ correspond to the two ortho and two meta deuterons. The 9.2, 9.2, and $62 \mathrm{~Hz}$ splittings of these peaks reflect the various values of $\left\langle Q_{i}\right\rangle$, namely $\left\langle Q_{o}\right\rangle=\left\langle Q_{m}\right\rangle=4.6 \mathrm{~Hz}$ and $\left\langle Q_{p}\right\rangle=31 \mathrm{~Hz}$. The similarity in $\left\langle Q_{o}\right\rangle$ and $\left\langle Q_{m}\right\rangle$ values is not expected on the basis of the estimates outlined above and warrants further comment.

As shown in Table 1, both liquid crystal NMR data and semiempirical calculations at the PM3 level indicate that $\beta_{p}=$ $180^{\circ}$. The additional benchmark needed for relating all of the residual couplings to structure is the value for at least one coupling constant, $Q_{i}$. Since $\beta_{p}$ is uniquely determined and $Q_{p}$ $=185 \mathrm{kHz}$ for the para deuteron in both sets of liquid crystal NMR data in Table 1 when $\eta$ is taken to be zero, it will be assumed that fixing $Q_{p}$ at $185 \mathrm{kHz}$ is a reasonable constraint. The projection on the top of Figure $3 \mathrm{a}$ indicates a value for $\left\langle Q_{p}\right\rangle$ of $31 \mathrm{~Hz}$, which is smaller than the $40 \mathrm{~Hz}$ value expected 
TABLE 1: Summary of Internal Angles and ${ }^{2} \mathrm{H}$ Quadrupolar Coupling Constants

\begin{tabular}{|c|c|c|c|c|c|c|c|}
\hline & \multicolumn{2}{|c|}{ liquid crystal } & \multicolumn{2}{|c|}{ electric field } & \multicolumn{3}{|c|}{ present study } \\
\hline & ZLI-1167 & $\mathrm{PCH}$ & $\beta_{i}$ fixed & $Q_{i}$ fixed & $\beta_{i}$ fixed & $Q_{i}$ fixed & PM3 \\
\hline$\beta_{o}$ & $58.3^{\circ}$ & $58.3^{\circ}$ & $58.3^{\circ}$ & $60.6^{\circ}$ & $58.3^{\circ}$ & $61.1^{\circ}$ & $60.8^{\circ}$ \\
\hline$\beta_{m}$ & $118.9^{\circ}$ & $118.9^{\circ}$ & $118.9^{\circ}$ & $120.2^{\circ}$ & $118.9^{\circ}$ & $119.8^{\circ}$ & $119.6^{\circ}$ \\
\hline$\beta_{p}$ & $180^{\circ}$ & $180^{\circ}$ & $180^{\circ}$ & $180^{\circ}$ & $180^{\circ}$ & $180^{\circ}$ & $180^{\circ}$ \\
\hline$Q_{o} / \mathrm{kHz}$ & 185 & 185 & 299 & 185 & 318 & 185 & 192 \\
\hline$Q_{m} / \mathrm{kHz}$ & 213 & 205 & 172 & 210 & 184 & 210 & 218 \\
\hline$Q_{p} / \mathrm{kHz}$ & 185 & 185 & 185 & 185 & 185 & 185 & 185 \\
\hline
\end{tabular}

on the basis of eqs $1-4$ with $Q_{p}=185 \mathrm{kHz}$ and $E_{\text {app }}=55$ $\mathrm{kV} / \mathrm{cm}$. This discrepancy between theory and experiment is most likely due to an inaccurate prediction of the alignment factor $2(p E / k T)^{2} / 15$ based on Onsager values for $p$ and $E$ in the condensed phase ${ }^{23}$ coupled with electric field inhomogeneity. The theoretical estimate does not include effects of interparticle correlations that would be naturally included in the Kirkwood approach to electrical polarization. ${ }^{24,25} \mathrm{In}$ addition, the decreased value of $2(p E / k T)^{2} / 15=\left\langle Q_{p}\right\rangle / Q_{p}=31 \mathrm{~Hz} / 185 \mathrm{kHz}=1.68 \times$ $10^{-4}$ in comparison to the theoretical value of $2.14 \times 10^{-4}$ may reflect electrode end effects, the overriding perturbation to electric field distortion when the diameter of circular plate electrodes does not greatly exceed their separation. Regardless of the source of this discrepancy, taking $Q_{p}=185 \mathrm{kHz}$ allows calculation of $2(p E / k T)^{2} / 15$ from the observed residual value of $\left\langle Q_{p}\right\rangle=31 \mathrm{~Hz}$. This factor can be used along with internal bond angles $\beta_{i}$ measured with liquid crystal techniques to determine $Q_{o}$ and $Q_{m}$ from the observed $\left\langle Q_{o}\right\rangle=\left\langle Q_{m}\right\rangle=4.6 \mathrm{~Hz}$ frequencies. These values are included in Table 1 along with a similar analysis of $1 \mathrm{D}$ electric field NMR data on $\mathrm{C}_{6} \mathrm{D}_{5} \mathrm{NO}_{2}$ obtained elsewhere.$^{26}$ Comparison of the two electric field data sets indicates that the ortho deuteron has a substantially larger $Q_{i}$ value than the meta and para sites, in disagreement with the liquid crystal data that display a larger $Q_{i}$ value for the meta site. The similarity between our electric field results and those of MacLean ${ }^{26}$ coupled with the anomalously large quadrupolar coupling constant for the meta $\mathrm{C}-\mathrm{D}$ bond suggests that either operation in pure liquids and/or in strong electric fields slightly modifies the structure of $\mathrm{C}_{6} \mathrm{D}_{5} \mathrm{NO}_{2}$. Taking $Q_{i}$ values from the liquid crystal NMR data and refitting both our data and that of MacLean $^{26}$ to determine new internal $\mathrm{C}-\mathrm{D}$ bond angles indicates that in both cases, both $\beta_{o}$ and $\beta_{m}$ increase by at least $1^{\circ}$, as shown in Table 1. Vindication of this refitting procedure is shown in the final column of Table 1, where bond angles determined with the Spartan PM3 semiempirical structure calculation are provided and combined with our experimental values for $\left\langle Q_{i}\right\rangle$ and $2(p E / k T)^{2} / 15$ to determine $Q_{o}, Q_{m}$, and $Q_{p}$ as 192,218 , and $185 \mathrm{kHz}$, respectively.

Comparison of the various $Q_{i}$ values in Table 1 suggest that the projection of the 2D spectrum in Figure 3a does reliably reproduce 1D NMR results. In Figure 3a and in all 2D spectra in this study, the horizontal axis corresponds to the Fourier transform of the free induction signal measured as a function of $t_{2}$ and the vertical axis represents the Fourier transform of the modulation of this signal as a function of $t_{1}$. Combination of rapid $\pi$ pulsing, where $\tau=3 \mathrm{~ms}$, with the double quantum filter in the indirect dimension $t_{1}=n \tau$ of Figure 2a successfully minimizes motional effects in the correlation spectrum in Figure 3a. The absolute values of the data are shown in this spectrum to emphasize the electric field induced splittings. Realization of the phase sensitive nature of this data allows one to consider only those peaks with either positive or negative intensity. Application of a shearing transform to either the positively or negatively phased data yields the spectrum in Figure 3b, correlating a completely isotropic chemical shift spectrum along

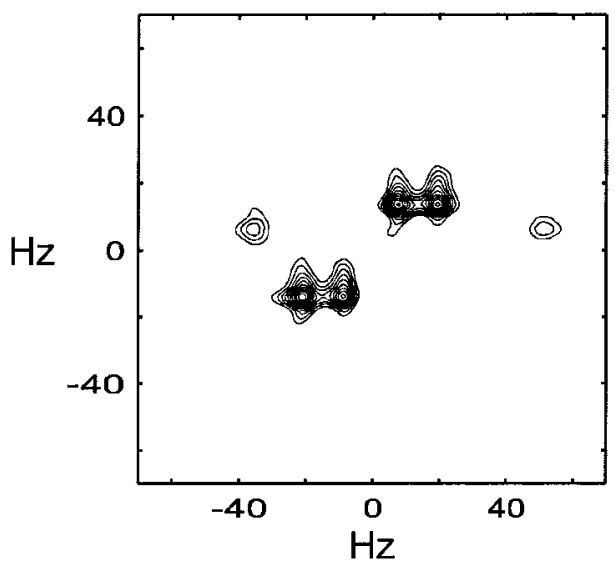

Figure 4. Two-dimensional ${ }^{2} \mathrm{H}$ NMR spectrum following Fourier transformation with respect to $t_{1}$ and $t_{2}$ of the data obtained using the sequence in Figure $2 \mathrm{~b}$ in a pulsed electric field. The projection onto the horizontal axis reflects the spectrum due to free precession in an electric field of $E_{\text {app }}=61 \mathrm{kV} / \mathrm{cm}$. Projection onto the indirect vertical axis gives the anticipated three-peak isotropic spectrum for $\mathrm{C}_{6} \mathrm{D}_{5} \mathrm{NO}_{2}$ since $E_{\text {app }}=0 \mathrm{kV} / \mathrm{cm}$ during the time $t_{1}$ in Figure $1 \mathrm{~b}$.

the vertical axis corresponding to $E_{\text {app }}=0 \mathrm{kV} / \mathrm{cm}$ with an anisotropic quadrupolar spectrum along the horizontal axis for $E_{\text {app }}=55 \mathrm{kV} / \mathrm{cm}$. This $2 \mathrm{D}$ spectrum represents one way of using an electric field to introduce anisotropy into a liquid sample, to extract useful residual couplings, and to retain the traditional high-resolution NMR spectrum for an isotropic liquid sample.

In actuality, the 2D spectrum in Figure $3 \mathrm{~b}$ is an artificial separation of isotropic and anisotropic spectral effects generated by mathematical manipulation of the measured spectrum in Figure 3a. In addition, the pulse sequence in Figure 2a used to produce these spectra does not exploit the full power of electrical orientation. Introducing a $61 \mathrm{kV} / \mathrm{cm}$ applied electric field as shown in Figure 2b produces the spectrum in Figure 4 for $\mathrm{C}_{6} \mathrm{D}_{5} \mathrm{NO}_{2}$ following Fourier transformation of the measured data with respect to $t_{1}$ and $t_{2}$. Clearly the directly detected dimension consists of six peaks, as seen by projecting the data in Figure 4 onto the horizontal axis.

Again this is due to a superposition of both chemical shift $\sigma_{i}$ and residual quadrupolar effects $\left\langle Q_{i}\right\rangle$ in an electric field. Also, the ratio of the residual quadrupolar coupling to the $185 \mathrm{kHz}$ quadrupolar coupling constant for the para deuteron can be used to find $2(p E / k T)^{2} / 15=\left\langle Q_{p}\right\rangle / Q_{p}=43.5 \mathrm{~Hz} / 185 \mathrm{kHz}=2.35 \times$ $10^{-4}$, which is again smaller than the $2(p E / k T)^{2} / 15=2.63 \times$ $10^{-4}$ expected for the applied $E_{\text {app }}=61 \mathrm{kV} / \mathrm{cm}$ field. Determination of the residual couplings for the ortho and meta sites as $\left\langle Q_{o}\right\rangle=\left\langle Q_{m}\right\rangle=6.5 \mathrm{~Hz}$ is consistent with the structure proposed above, since the ratio $\left\langle Q_{p}\right\rangle\left\langle\left\langle Q_{o}\right\rangle=6.7\right.$ is conserved between DC and pulsed electric field results. The indirectly detected dimension along the vertical axis of Figure 4 contains only three peaks, reflecting evolution of the magnetization under $\sigma_{i}$ during the period $t_{1}$ as anticipated with $E_{\text {app }}=0 \mathrm{kV} / \mathrm{cm}$.

Although the data in Figure 4 does provide spectral simplification by removing the effect of quadrupolar coupling in the 


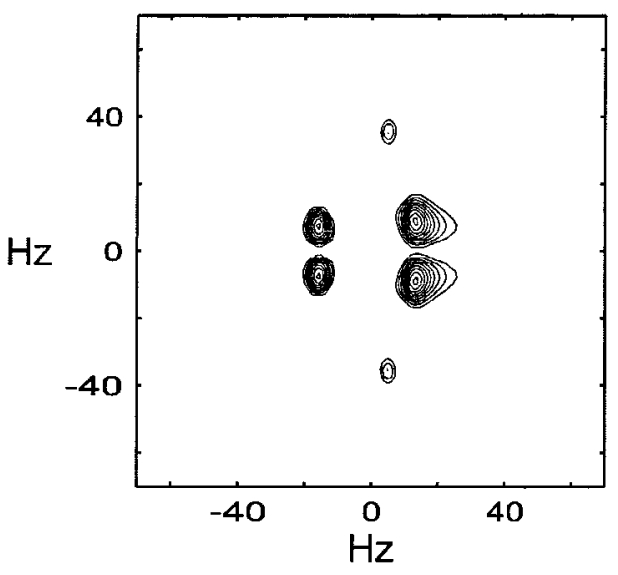

Figure 5. Two-dimensional ${ }^{2} \mathrm{H}$ NMR spectrum correlating directly detected isotropic effects with indirectly detected anisotropic spectral effects. A pulsed electric field of $E_{\text {app }}=58 \mathrm{kV} / \mathrm{cm}$ is used as shown in Figure $2 \mathrm{c}$ to produce this spectrum.

indirect dimension, it displays information only as useful as the correlation shown in Figure 3a for a continuous DC field. The 2D spectrum in Figure 5 was recorded using a $58 \mathrm{kV} / \mathrm{cm}$ pulsed electric field with $\tau=2 \mathrm{~ms}, m=19$, and $p=20$ in the sequence in Figure 2c. Projection onto the horizontal axis yields the anticipated three-peak isotropic spectrum representative of $\mathrm{C}_{6} \mathrm{D}_{5} \mathrm{NO}_{2}$, while projection along the indirect vertical axis yields solely the electrically induced quadrupolar splittings. Again, the alignment factor is smaller than the anticipated theoretical limit set at $2.14 \times 10^{-4}$ for $E_{\text {app }}=58 \mathrm{kV} / \mathrm{cm}$. Here the $65.8 \mathrm{~Hz}$ splitting for the para deuteron gives $2(p E / k T)^{2} / 15=32.9 \mathrm{~Hz} /$ $185 \mathrm{kHz}=1.77 \times 10^{-4}$. It is important to note that it is difficult to accurately determine the center of gravity of the contour shapes for the ortho and meta sites in Figure 5. Taking the average of the maximum and minimum splitting gives $\left\langle Q_{o}\right\rangle=$ $\left\langle Q_{m}\right\rangle=4.9 \mathrm{~Hz}$. Thus, the ratio $\left\langle Q_{p}\right\rangle /\left\langle Q_{o}\right\rangle=6.7$ is again conserved and can be used to demonstrate that the pulsed electric field does not appreciably change the structure of $\mathrm{C}_{6} \mathrm{D}_{5} \mathrm{NO}_{2}$ from that anticipated for a continuous electric field. Finally, the apparent loss of intensity of the para deuteron peaks reveals one of the shortcomings of this sequence, namely that the peak intensities in the final spectrum depended on $\left\langle Q_{i}\right\rangle$ and on the rephasing period set by $m$. Because the rephasing depends on $\left\langle Q_{i}\right\rangle$, which varies from site to site within a molecule, resulting spectral peak intensities may not accurately reflect site abundance. For molecules having a limited number of sites, such as $\mathrm{C}_{6} \mathrm{D}_{5} \mathrm{NO}_{2}$, it is possible to choose $m$ so that all quadrupolar sites are observed. However for larger, more complicated molecules it may be necessary to vary $m$ as a third dimension in the sequence.

\section{Conclusion}

Knowledge of the time scale and properties of electrical convection in solution permit design of multiple rf pulse sequences interleaved with electric impulses to provide correlations between isotropic and anisotropic spectral effects. Even though the test system used here displays a rather simple 1D NMR spectrum, these sequences are completely applicable to any electrically aligned system. The similarity in $\left\langle Q_{p}\right\rangle /\left\langle Q_{o}\right\rangle$ ratios in both DC and pulsed electric field experiments demonstrates that voltage gating does not require an alternative interpretation of the measured data. These 2D measurements combined with earlier 1D NMR experiments strongly suggest that the molecular structure of $\mathrm{C}_{6} \mathrm{D}_{5} \mathrm{NO}_{2}$ in pure solution and in an electric field is slightly different than that deduced in liquid crystal solvent.

Additionally, these sequences are not restricted to just ${ }^{2} \mathrm{H}$ nuclei. They should equally apply to molecules having any $I>$ $1 / 2$ isotopes containing nonzero quadrupolar interactions as well as dipolar coupled $I=1 / 2$ systems. Admittedly, the usefulness of separation of electrically dependent data from the usual liquids spectrum reflecting an isotropic environment is not important for simple test samples like $\mathrm{C}_{6} \mathrm{D}_{5} \mathrm{NO}_{2}$. However, eventual exploration of complicated organic and biological macromolecules having congested 1D NMR spectra will be necessarily complicated by application of an electric field. It is in this arena where pulse sequences like those in Figure 2 will be indispensable for refinement of molecular structure by NMR.

Acknowledgment. The authors are indebted to both Erwin Hahn and Jeff Reimer for stimulating conversation and continued encouragement throughout the course of this work. This work was supported by the National Science Foundation under Grant No. CHE-9504655, the Packard Foundation, and UC Davis.

\section{References and Notes}

(1) See, for example: Chem. Rev. 1991, 91.

(2) Protein data bank at Brookhaven National Laboratory; Web site: pdb.pdb.bnl.gov.

(3) Wuthrich, K. NMR of Proteins and Nucleic Acids; John Wiley and Sons: New York, 1986.

(4) Ernst, R. R.; Bodenhausen, G.; Wokaun, A. Principles of Nuclear Magnetic Resonance in One and Two Dimensions; Oxford University Press: New York, 1997.

(5) The ${ }^{1} \mathrm{H}-{ }^{1} \mathrm{H}$ dipolar coupling for $r=5 \AA$ is $\omega_{\mathrm{D}} / 2 \pi=4.8 \mathrm{kHz}$ giving $1 / T_{1} \mathrm{NOE} \approx\left(\omega_{\mathrm{D}} / 2 \pi\right)^{2} \tau_{\mathrm{c}}=4.8 \mathrm{~Hz}$ for $\tau_{\mathrm{c}}=1 \mu \mathrm{s}$.

(6) Prosser, R. S.; Hunt, S. A.; Vold, R. R. J. Magn. Reson. Ser. B 1995, 109, 109.

(7) Haeberlen, U. High Resolution NMR in Solids: Selective Averaging; In Advances in Magnetic Resonance, Sup. 1; Waugh, J., Ed.; Academic Press: New York, 1976.

(8) Gullion, T.; Schaefer, J. J. Magn. Reson. 1989, 81, 196.

(9) Slichter, C. P. Principles of Magnetic Resonance; SpringerVerlag: New York, 1990.

(10) Tolman, J. R.; Flanagan, J. M.; Kennedy, M. A.; Prestegard, J. H. Proc. Natl. Acad. Sci. 1995, 92, 9279.

(11) Tjandra, N.; Bax, A. J. Magn. Reson. 1997, 124, 512.

(12) Kung, H. C.; Wang, K. Y.; Goljer, I.; Bolton, P. H. J. Magn. Reson. Ser. B 1995, 109, 323.

(13) Hansen, M. R.; Mueller, L.; Pardi, A. Nature Struc. Biol. 1998, 5, 1065.

(14) Bothner-By, A. A.; Domaille, P. J.; Gayathri, C. J. Am. Chem. Soc. 1981, 103, 5602 .

(15) Sears, R. E. J.; Hahn, E. L. J. Chem. Phys. 1966, 45, 2753.

(16) Hilbers, C. W.; MacLean, C. Chem. Phys. Lett. 1968, 2, 445.

(17) Deutch, J. M.; Waugh, J. S. J. Chem. Phys. 1965, 43, 4366.

(18) Biemond, J. Dielectric Alignment of Molecules in NMR; Rodopi N.V.: Amsterdam, 1975.

(19) Riley, S. A.; Augustine, M. P. J. Magn. Reson. In press.

(20) Catalano, D.; Forte, C.; Veracini, C. A. J. Magn. Reson. 1984, 60, 190.

(21) Onsager, L. J. Am. Chem. Soc. 1936, 58, 1486.

(22) Carr, H. Y.; Purcell, E. M. Phys. Rev. 1954, 94, 630.

(23) Plantenga, T. M.; De Kanter, F. J. J.; Bulsink, H.; MacLean, C. Chem. Phys. 1982, 65, 77.

(24) Kirkwood, J. J. Chem. Phys. 1939, 7, 911.

(25) Biemond, J.; MacLean, C. Mol. Phys. 1974, 28, 571.

(26) Van Zijl, P. C. M.; Ruessink, B. H.; Bulthuis, J.; MacLean, C. Acc. Chem. Res. 1984, 17, 172. 